\title{
Effect of Substrate Chemistry on Prenucleation
}

\begin{abstract}
C.M. FANG, H. MEN, and Z. FAN
Understanding atomic ordering in a metallic liquid adjacent to a solid substrate is of both scientific and technological importance. In this study, we used ab initio molecular dynamics (MD) simulations to investigate systematically the effect of substrate chemistry on atomic ordering in the liquid adjacent to artificially created substrates that have the same crystal structure as the solid phase upon solidification but different chemical characteristics. We found that for a given liquid, an attractive chemical interaction (negative heat of mixing) between the liquid and the substrate strengthens atomic ordering in the liquid at the interface, while a repulsive interaction (positive heat of mixing) weakens atomic ordering. It is realized that although both structure and chemistry of the substrate affect atomic ordering in the liquid adjacent to the liquid/substrate interface, the structural effect dominates the atomic ordering process, while the chemical effect is but a secondary factor and affects atomic ordering through either strengthening or weakening the structural templating. Such knowledge of atomic ordering may help us to understand both prenucleation and heterogeneous nucleation, and to develop more effective approaches to grain refinement during solidification through effective manipulation of the interplay between structural and chemical effects of the substrate on atomic ordering in the liquid adjacent to the interface.
\end{abstract}

https://doi.org/10.1007/s11661-018-4882-y

(C) The Author(s) 2018

\section{INTRODUCTION}

UNDERSTANDING atomic arrangement in the liquid adjacent to a liquid/substrate interface is of fundamental importance to both scientific research and technological development, such as heterogeneous nucleation, catalysis, lubrication, and so on ${ }^{[1]}$ However, our current knowledge of the subject is still very limited mainly due to the lack of appropriate experimental approaches. ${ }^{[2]}$ The epitaxial nucleation model ${ }^{[3]}$ offers an atomistic mechanism for heterogeneous nucleation of solidification, in which the lattice of the substrate surface provides structural templating for the formation of the solid phase. Recent research findings ${ }^{[4]}$ seem to support this atomistic mechanism. Both experimental observations $^{[4,5]}$ and atomistic simulations ${ }^{[6-8]}$ have revealed that the atoms in the liquid adjacent to a crystalline substrate become layered within a couple of nanometres away from the interface, and such interfacial layers may exhibit substantial in-plane atomic ordering within the first 2 to 3 atomic layers. Similarly, such atomic ordering has also been observed in the liquid adjacent to its own surface. ${ }^{[9-12]}$ Such atomic

C.M. FANG, H. MEN and Z. FAN are with the BCAST, Brunel University London, Uxbridge, Middlesex, UB8 3PH, UK. Contact e-mail: Zhongyun.Fan@brunel.ac.uk

Manuscript submitted May 11, 2018.

Article published online September 27, 2018 layering has been attributed to the "hard wall" effect of the substrate, ${ }^{[10]}$ and the in-plane atomic ordering is induced by the lattice of the substrate. ${ }^{[5]}$ This suggests that such atomic ordering in the liquid is closely related to the physical and chemical nature of the substrate for a given liquid. ${ }^{[13]}$

The pronounced atomic ordering in the liquid adjacent to the liquid/substrate interface at temperatures above the nucleation temperature has been referred to as prenucleation, which may have a significant influence on the subsequent heterogeneous nucleation process during solidification. ${ }^{[14]}$ The prenucleation induced by the substrate is manifested by the atomic layering normal to the interface, in-plane atomic ordering parallel to the interface and the formation of a 2-dimensional (2D) ordered structure at the interface. Usually, the ordered structure at the interface continues the lattice of the substrate through a structural templating mechanism. ${ }^{[14]}$ It has been reported that atomic ordering at the interface could be affected by structural properties of the substrate, such as crystal structure ${ }^{[7]}$ surface orientation, ${ }^{[7,15]}$ and lattice parameter. ${ }^{[6]}$ With classical MD simulations, our previous study ${ }^{[6]}$ revealed that the lattice misfit between the substrate and the solid phase (corresponding to the liquid upon solidification) has a significant effect on the in-plane atomic ordering and the formation of the interfacial 2D ordered structures in the liquid, where the effect of substrate chemistry on atomic ordering can be excluded by choosing $\mathrm{Al}$ atoms as the substrate. However, it is not clear how the chemical 
interaction between the liquid and the substrate affects the prenucleation phenomenon.

$A b$ initio MD simulation is particularly suitable for investigating the chemical effect of the substrate on prenucleation since it is parameter-free and can explore any element of interest. Using $a b$ initio MD simulations, Wang et al. ${ }^{[16]}$ observed an fcc (face-centered cubic)-like ordering in the liquid $\mathrm{Al}$ adjacent to the Ti-terminated $\mathrm{TiB}_{2}$ surface, but a disordered structure at the B-terminated $\mathrm{TiB}_{2}$ surface. Their results also suggest that the growth of the $\alpha$-Al was frustrated by the lattice misfit between the bulk solid $\mathrm{Al}$ and the $\mathrm{TiB}_{2}$ substrate, at a small undercooling. This variation in atomic arrangements in the liquid adjacent to the Ti- or B-terminated surface of the $\mathrm{TiB}_{2}$ substrate implies that the chemical interaction between the liquid $\mathrm{Al}$ and the $\mathrm{TiB}_{2}$ surface has a strong influence on atomic ordering in the liquid at the interface in addition to the structural effect. Therefore, it is beneficial to investigate the effect of substrate chemistry on the atomic ordering at the interface, preferably exclusive of the structure effect.

The pinned substrate approach has been validated to investigate systematically the effect of the structural properties (crystal structure, surface orientation, and lattice misfit) of the substrate on the atomic ordering at the interface. ${ }^{[14]}$ By pinning the substrate atoms with varied lattice parameter and choosing the same element for both the substrate and the liquid, we clarified the effect of the lattice misfit on atomic ordering in the liquid at the interface. $^{[14]}$ In this way, all MD simulations were conducted for systems with the same chemical interaction between the liquid and the substrate. Therefore, only the structural effect on atomic ordering in the liquid was assessed without the interference of the chemical effect. This approach provides a unique method to distinguish the structural effect of the substrate from the chemical effect, and therefore can be used to assess independently the effects of chemical and structural properties of the substrate on the atomic ordering at the interface. The chemical effect of the substrate can be investigated with the pinned substrate approach by varying the chemistry of the substrate while its structural effect is excluded. As suggested by A. Takeuchi and A. Inoue, ${ }^{[17]}$ a negative heat of mixing $\left(\Delta H^{\mathrm{mix}}\right)$ indicates an attractive interaction between the atoms of the substrate and those of the liquid, and a positive value indicates a repulsive interaction. Thus, the chemical property of the substrate in the simulation system can be quantified by the $\Delta H^{\text {mix }}$ between the substrate and liquid atoms.

In this study, we aim to investigate the effect of substrate chemistry and temperature on prenucleation by ab initio MD simulations while excluding the structure effect. We validate first the pinned substrate approach by comparing with the case of a relaxed substrate. The effect of the substrate chemistry on the atomic ordering in the liquid adjacent to the liquid/substrate interface is then investigated systematically. The electronic density distribution at the interface is also examined to understand the nature of the chemical effect. The effect of the substrate chemistry on prenucleation and its implication for heterogeneous nucleation are discussed.

\section{SIMULATION APPROACH}

\section{A. Setting up Simulation Systems}

For simplicity, both the liquid and the substrate in the simulation system were chosen to be pure metals. While the liquid was fixed to be $\mathrm{Al}$, the substrate metals were selected according to the following two criteria: (1) the substrate metal has a closely packed crystal plane which matches closely the atomic arrangement of $\{111\}_{\mathrm{Al}}$; (2) the substrate metal has a strong chemical interaction with liquid $\mathrm{Al}$, being either strongly attractive or strongly repulsive. In other words, the substrate metal should have a heat of mixing $\left(\Delta H^{\mathrm{mix}}\right)$ with liquid $\mathrm{Al}$ that is either strongly negative or strongly positive. Based on these criteria, four elements, $\mathrm{Ag}, \mathrm{W}, \mathrm{Al}$, and $\mathrm{Cd}$, were selected to be the substrate metals to provide a wide range of chemical interactions. The crystallographic and thermodynamic data for the substrate metals are summarized in Table I. $\Delta H^{\text {mix }}$ is $-4.0,-2.0,0.0$ and 3.0 $\mathrm{kJ} / \mathrm{mol}$, respectively, for the $\mathrm{Al} / \mathrm{Ag}, \mathrm{Al} / \mathrm{W}, \mathrm{Al} / \mathrm{Al}$, and $\mathrm{Al} / \mathrm{Cd}$ (liquid/substrate) systems. ${ }^{[\mathrm{P}]}$ The $\mathrm{Ag}$ and $\mathrm{W}$ substrates have an attractive interaction with liquid Al, while the $\mathrm{Cd}$ substrate has a repulsive interaction with liquid Al. ${ }^{[17,18]}$ This agrees well with the information provided by their binary phase diagrams: both Al-Ag and Al-W are binary compound-forming systems, ${ }^{[19-21]}$ and the Cd-Al system has a large miscibility gap. ${ }^{[20]}$ The interaction between the $\mathrm{Al}$ substrate and liquid $\mathrm{Al}$ is neutral, and therefore the $\mathrm{Al} / \mathrm{Al}$ system was used as a reference to assess the chemical effect of the substrate on the atomic ordering in the liquid at the interface.

In order to assess the chemical effect without interference from the structural effect, we have pinned substrate atoms at the fcc lattice positions with a constant lattice parameter of $a=4.12 \AA$, which is the calculated lattice parameter of solid $\mathrm{Al}$ at its melting point, $933 \mathrm{~K} .{ }^{[3]}$ In other words, the artificially constructed substrates always have the crystal structure of Al, but have different chemical characteristics. For example, the constructed Ag substrate has an fcc crystal structure with a lattice parameter of $a=4.12 \AA$, but has the chemical nature of Ag. This allows us to assess the chemical effect of the substrate on the prenucleation for the simulation systems with a constant lattice misfit of $f$ $=0$ pct, ${ }^{[22]}$ i.e., the structural effect of the substrate was excluded completely. The validity of the pinned substrate approach was checked by comparing the atomic ordering in the liquid at the interface in the $\mathrm{Al} / \mathrm{Ag}$ system with a pinned substrate and a relaxed substrate. Another advantage of this pinned substrate approach is to allow elements with a lower melting point than $\mathrm{Al}$ (e.g., Cd) to act as substrates for simulations.

To justify the configuration of the substrates, the lattice parameter of bulk solids was calculated with $a b$ initio structural optimizations at the ground state. The calculated lattice parameter is 4.039, 4.151, 3.174, and $4.023 \AA$, respectively, for fcc Al, fcc Ag, bcc (body-centered cubic) W, and fcc W. This is in good agreement with experimental values in the literature: ${ }^{[18]}$ $4.050 \AA, 4.086 \AA$ and $3.165 \AA$, respectively, for fcc $\mathrm{Al}$, fcc Ag, and bcc W at room temperature. It should be 
Table I. Summary of Crystallographic and Thermodynamic Data for the Elements of Al, Ag, W, and Cd

\begin{tabular}{|c|c|c|c|c|c|}
\hline Element & $\begin{array}{l}\text { Crystal } \\
\text { Structure }\end{array}$ & Lattice Parameter $^{\mathrm{b}}(\AA)^{[18]}$ & Orientation Relationship $^{c}$ & Lattice Misfit $^{\mathrm{d}}$ (Pct) & $\Delta H^{\operatorname{mix~e}}(\mathrm{kJ} / \mathrm{mol})^{[17]}$ \\
\hline $\mathrm{Al}$ & fcc & $a=4.05$ & $\langle 110\rangle\{111\}_{\mathrm{Al}} / /\langle 110\rangle\{111\}_{\mathrm{Al}}$ & 0.0 & 0.0 \\
\hline $\mathrm{Ag}$ & fcc & $a=4.09$ & $\langle 110\rangle\{111\}_{\mathrm{Al}} / /\langle 110\rangle\{111\}_{\mathrm{Ag}}$ & -0.8 & -4.0 \\
\hline W & $\mathrm{fcc}^{\mathrm{a}}$ & $a=4.02$ & $\langle 110\rangle\{111\}_{\mathrm{Al}} / /\langle 110\rangle\{111\}_{\mathrm{W}}$ & +0.7 & -2.0 \\
\hline $\mathrm{Cd}$ & hcp & $\begin{array}{l}a=2.98 \\
c=5.62\end{array}$ & $\langle 110\rangle\{111\}_{\mathrm{Al}} / /[11-2](0001)_{\mathrm{Cd}}$ & -3.7 & +3.0 \\
\hline
\end{tabular}

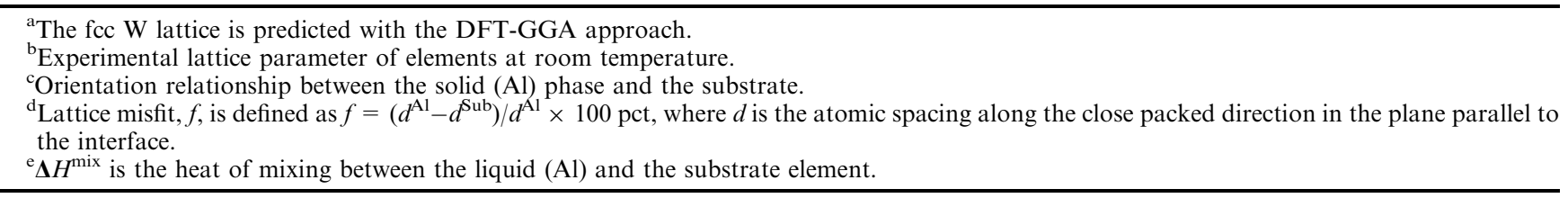

pointed out that fcc $\mathrm{W}$ is predicted to be metastable, and the energy difference is $+0.486 \mathrm{eV} /$ atom between fcc W and bcc W at the ground state. The calculated lattice parameters for hop (hexagonal close packed) $\mathrm{Cd}, a=$ $3.026 \AA$ and $c=5.681 \AA$, are slightly overestimated, compared to experimental values of $a=2.978 \AA$ and $c$ $=5.617 \AA{ }^{[18]}$ Such overestimation is not unusual for the DFT-GGA approach. ${ }^{[23]}$ The calculated lattice misfit between the substrate and fcc Al is relatively small, less than 3.7 pct (Table I). This suggests that the electronic structure of the substrate atoms will not be distorted substantially with the pinned substrate when they adopt the lattice parameter of bulk Al.

In our simulations, the periodic boundary conditions were employed. We built a hexagonal supercell based on the conventional unit cell of Al. The hexagonal supercell has the dimensions of $5 a_{\mathrm{Al}(111)} \times 5 a_{\mathrm{Al}(111)} \times 7 c_{\mathrm{Al}(111)}$, here $a_{\mathrm{Al}(111)}=(\sqrt{ } 2 / 2) a(\mathrm{Al}), c_{\mathrm{Al}(111)}=\sqrt{3} a(\mathrm{Al})$, and $a(\mathrm{Al})$ $(=4.12 \mathrm{~A})$ being the lattice parameter of $\mathrm{Al}$ at its melting point. ${ }^{[3,6]}$ In this way the built supercell has lengths of the axis being $a=b=14.57 \AA, c=49.95 \AA$; $\alpha=90 \mathrm{deg}, \beta=120 \mathrm{deg}$, and $\gamma=90 \mathrm{deg}$. This supercell contains 4 and $17\{111\}$ planes in thickness, respectively, for the substrate and initial configuration of liquid Al, with 100 substrate atoms and 425 liquid $\mathrm{Al}$ atoms. We believe that the supercell is adequate in thickness for both the substrate and the liquid $\mathrm{Al}$ for the purpose of this investigation.

\section{B. Computation Details}

All first principle calculations were performed using the code VASP (Vienna $a b$ initio Simulation Package). ${ }^{[24,25]}$ Ab initio density functional theory (DFT) within the projector-augmented wave (PAW) framework was employed. ${ }^{[26,27]}$ The exchange and correlation terms were described using the generalized gradient approximation (GGA), formulated by Perdew, Burke, and Ernzerhof (PBE) ${ }^{[28]}$ which has been proven to well describe transition metals and their intermetallics or precipitates. ${ }^{[29,30]}$ The atomic electronic configurations in pseudo-potentials are $\mathrm{Al}\left([\mathrm{Ne}] 3 \mathrm{~s}^{2} 3 \mathrm{p}^{1}\right), \mathrm{Ag}([\mathrm{Kr}]$ $\left.4 \mathrm{~d}^{10} 5 \mathrm{~s}^{1} 5 \mathrm{p}^{0}\right), \mathrm{Cd}\left([\mathrm{Kr}] 4 \mathrm{~d}^{10} 5 \mathrm{~s}^{2} 5 \mathrm{p}^{0}\right)$, and $\mathrm{W}$ ([Xe] $\left.4 f^{14} 5 d^{4} 6 s^{2} 5 p^{0}\right)$. For total energy and electronic structure calculations, the cut-off energies of $400.0 \mathrm{eV}$ for the wave functions and $550.0 \mathrm{eV}$ for the augmentation functions are used to describe the transition metals. The cut-off energies are noticeably higher than the default energies for the metals. The electronic wave functions were sampled on $20 \times 20 \times 20,24 \times 24 \times 24$, and $24 \times 24 \times 20 k$-mesh, respectively, which correspond to 220,364 , and $1560 k$-points in the irreducible Brillouin zones (BZ) of the conventional unit cells of the fcc Al, Ag, and W; bcc W; and hep Cd. ${ }^{[31]}$ The ab initio MD simulation is based on the finite-temperature density-functional theory of the one-electron states, the exact energy minimization and calculation of the exact Hellmann-Feynman forces after each MD step using preconditioned conjugate techniques, and the Nosé dynamics for generating a canonical NVT ensemble. ${ }^{[24,25]}$ The present method allows variable fractional occupation numbers, and therefore, it works well for metallic systems. Only $\Gamma$-point in the BZ with cut-off energy of $200 \mathrm{eV}$ was employed to perform ab initio MD simulations for the large supercells described above, in order to balance the requirements of large computational costs and accuracy of the simulated results.

Liquid $\mathrm{Al}$ was generated by equilibrating the $\mathrm{Al}$ atoms at $3000 \mathrm{~K}$ for about 6000 to 8000 time steps (1.5 fs/step), or $\sim 10$ picoseconds (ps), and then cooled to the designed temperature. The liquid $\mathrm{Al}$ was brought to contact with the substrate for building the L-Al/S-M interface systems $(\mathrm{M}=\mathrm{Ag}, \mathrm{Mo}, \mathrm{Al}$, and $\mathrm{Cd})$. The starting interface systems were equilibrated at the designed temperature for 6000 to 8000 steps. To obtain a meaningful statistical analysis, ${ }^{[24,32,33]}$ we employed the time-averaged method by taking samples at an interval of 75 fs over 3 to 5 ps. This approach has been validated by testing the results for varied simulation systems with different thickness.

\section{Characterization of Atomic Ordering}

Atomic density profile, $\rho(z)$, and in-plane order parameter, $S(z)$, are usually used to characterize the atomic ordering along the direction normal to the interface (layering) and in the $x-y$ plane parallel to the interface (in-plane ordering), respectively. ${ }^{[6,7,22]}$ The density profile is defined as ${ }^{[T]}$ 


$$
\rho(z)=\left\langle N_{\mathrm{z}}(t)\right\rangle /\left(L_{\mathrm{x}} L_{\mathrm{y}} \Delta z\right),
$$

where $L_{\mathrm{x}}$ and $L_{\mathrm{y}}$ are the $x$ and $y$ dimensions of the cell, respectively, and $z$ the dimension perpendicular to the interface, and $\Delta z$ is the bin width ( $=0.2 \AA$ here), and $N_{z}(t)$ is the number of atoms between $z-(\Delta z / 2)$ and $z+(\Delta z / 2)$ at time $t .\left\langle N_{z}(t)>\right.$ indicates a time-averaged number of atoms. Therefore, the atomic density profile value $(\rho(z))$ at peak position is equal to 0.68 atoms $/ \AA^{3}$ for the pinned substrate. ${ }^{[7,22]}$ The in-plane order parameter is defined as ${ }^{[34]}$

$$
S(z)=\left[\sum \exp \left(i \boldsymbol{Q} \cdot \boldsymbol{r}_{\mathrm{j}}\right)\right]^{2} / N_{\mathrm{z}}^{2},
$$

where the summation is over all atoms within a given bin of width $\Delta z$, and $\boldsymbol{Q}$ is the reciprocal lattice vector, $\boldsymbol{r}_{\mathrm{j}}$ is the Cartesian coordinates of the jth atom, and $N_{\mathrm{z}}$ is the number of atoms in the bin (layer). The in-plane ordering is an average over the local order parameter of the atoms in the individual layer in the liquid at the interface.

\section{RESULTS}

\section{A. Pinned Substrate Vs Relaxed Substrate}

To validate our simulation approach, we first examined the effect of pinned and relaxed substrates on atomic ordering in the liquid adjacent to the interface. Figure 1 shows snapshots of part of the simulation systems with the interface of liquid $\mathrm{Al}$ and pinned/ relaxed $\mathrm{Ag}$ substrates equilibrated at $1000 \mathrm{~K}$. The melting temperature of $\mathrm{Ag}, 1235 \mathrm{~K}$, is higher than that of Al, and thus the relaxed Ag substrate remains solid during the equilibration at $1000 \mathrm{~K}$. Both systems exhibit a distinct layered structure in the liquid adjacent to the interface. The density profiles, $\rho(z)$, of the systems with pinned or relaxed Ag substrate are plotted in Figure 2(a) as a function of distance $z$ from the interface. The

(a) Pinned substrate

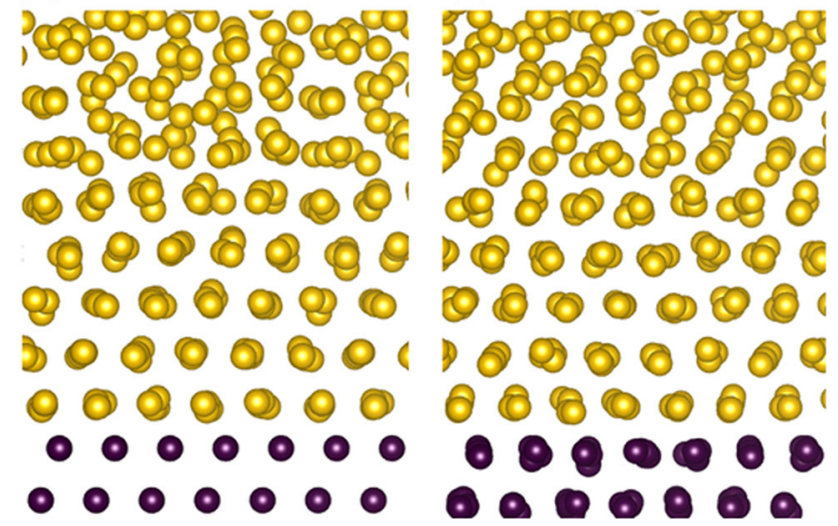

Fig. 1-Snapshots of the simulation systems with the liquid $\mathrm{Al}$ and the $\mathrm{Ag}$ substrate equilibrated at $1000 \mathrm{~K}$, where the substrate atoms are $(a)$ pinned or $(b)$ relaxed. The dark (violet online) and light (gold online) spheres represent the substrate and liquid atoms, respectively (Color figure online). dashed and solid lines represent the density profiles for the systems with the pinned and relaxed substrates,

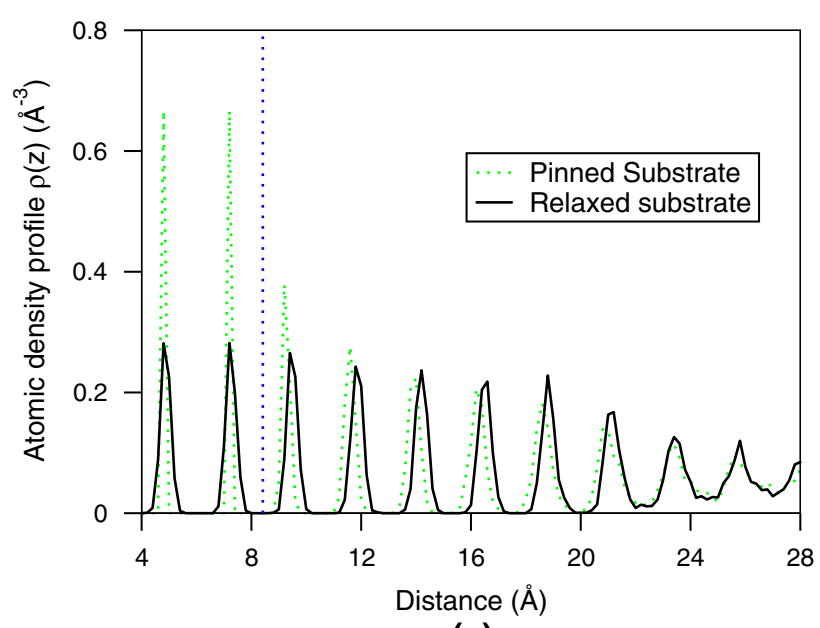

(a)

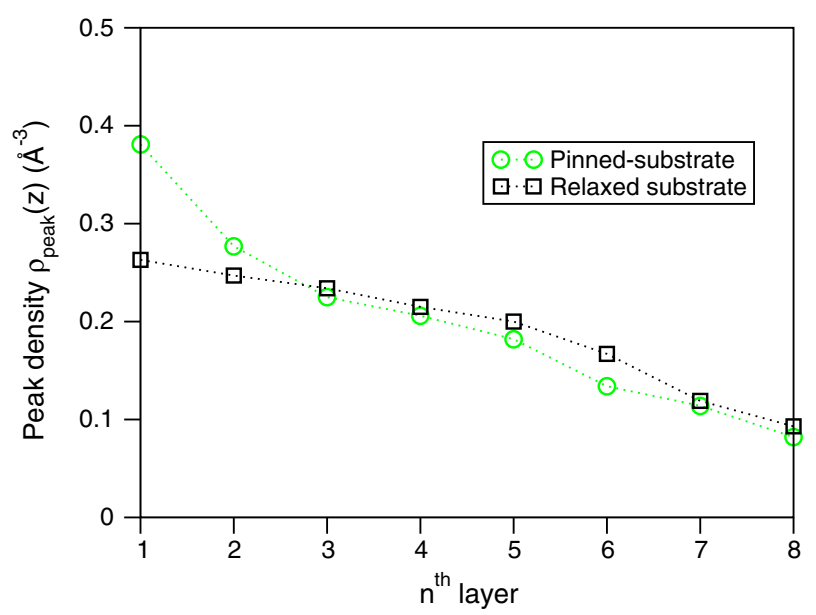

(b)

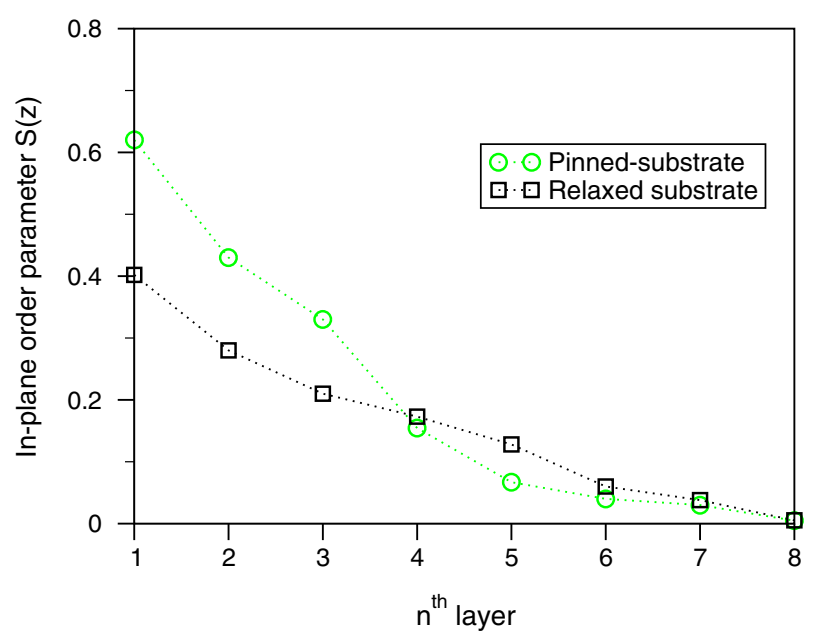

(c)

Fig. 2 - (a) Atomic density profiles, $\rho(z)$; (b) peak density, $\rho_{\text {peak }}$; and (c) in-plane order parameter, $S(\mathrm{z})$, of the liquid $\mathrm{Al}$ adjacent to the Ag substrate equilibrated at $1000 \mathrm{~K}$ as a function of the distance $z$ or atomic layers from the interface, where the substrate atoms are either pinned or relaxed (Color figure online). 
respectively. Figure 2(a) suggests that the $\mathrm{Al}$ atoms in the liquid adjacent to the interface exhibit similar layering behavior for both cases although the density of the first peak for the relaxed substrate is lower than that of the pinned substrate (Figure 2(b)). Similarly, the $\mathrm{Al}$ atoms in the liquid adjacent to the interface exhibit pronounced in-plane ordering for both cases although the $S(z)$ of the first 3 interfacial layers for the relaxed substrate is slightly lower than that of the corresponding layers for the pinned substrate (Figure 2(c)). The reduced $\rho_{\text {peak }}$ and $S(z)$ for the systems with a relaxed substrate can be attributed to the increased thermal vibration of the substrate atoms at their equilibrium positions, which decreases the effectiveness of the substrate for structural templating, leading to lower values for $\rho_{\text {peak }}$ and $S(z)$ for the first few layers, compared to those for the pinned substrate. To sum up, although the relaxed substrate does change the atomic ordering in the liquid at the interface compared with the pinned substrate, such changes do not affect the general trend of atomic ordering, and consequently only pinned substrates are considered afterwards in this study.

\section{B. Effect of Substrate Chemistry on Atomic Layering in the Liquid}

We investigated the effect of substrate chemistry on atomic layering in the liquid adjacent to the interface. Figure 3 shows snapshots of the simulation systems with pinned $\mathrm{Ag}, \mathrm{Al}$, and $\mathrm{Cd}$ substrates equilibrated at $1000 \mathrm{~K}$. The dark (violet online) and light (gold online) spheres represent the substrate and liquid atoms, respectively, and the solid (orange online) lines mark the interface between the substrates and the liquid. Atoms in the liquid adjacent to the interface exhibit stronger atomic layering with the Ag substrate (Figure 3(a)) and weaker atomic layering with the $\mathrm{Cd}$ substrate (Figure 3(c)), compared with that with an Al substrate (Figure 3(b)). To characterize the layering, the atomic density profile, $\rho(z)$, of the liquid is plotted in Figure 4 as a function of distance $z$ for the systems with different pinned substrates equilibrated at $1000 \mathrm{~K}$. The 1 st dotted line marks the liquid/substrate interface, and the 2nd dotted line marks the peak position of the 1st layer of liquid in the system with the Al substrate. Figure 4 reveals the following facts: (1) the $\mathrm{Al}$ atoms in the liquid adjacent to the interface exhibit higher peak density with the $\mathrm{Ag}$ substrate and lower peak density with the Cd substrate, compared to that with the Al substrate; (2) the atomic layering persists for 8, 6, and 5 atomic layers in the liquid for the $\mathrm{Ag}, \mathrm{Al}$, and $\mathrm{Cd}$ substrates, respectively; and (3) the peak position of the 1st layer shifts closer to the interface with the $\mathrm{Ag}$ substrate and further away from the interface with the Cd substrate, compared with that with the Al substrate.

To quantify the effect of substrate chemistry, the peak density $\left(\rho(z)_{\mathrm{L} 1}\right)$ interlayer spacing $\left(d_{\mathrm{L} 1}\right)$ and in-plane ordering $\left(S(z)_{\mathrm{L} 1}\right)$ of the first layer, and the number of layers $\left(n_{\mathrm{L}}\right)$ in the liquid adjacent to the interface are plotted in Figure 5 as a function of heat of mixing, $\Delta H^{\mathrm{mix}}$, at $1000 \mathrm{~K}$. The data from the simulation system with a pinned $\mathrm{W}$ substrate are also included, where $\mathrm{W}$ has a heat of mixing of $-2.0 \mathrm{~kJ} / \mathrm{mol}$ with Al. With decreasing $\Delta H^{\text {mix }}$ from positive to negative, $\rho(z)_{\mathrm{L} 1}$, $S(z)_{\mathrm{L} 1}$, and $n_{\mathrm{L}}$ increase linearly, while the interlayer spacing, $d_{\mathrm{L}}$, between the substrate and the 1st layer in the liquid decreases linearly. Therefore, it can be concluded that an attractive interaction between the liquid and the substrate enhances atomic ordering at the interface, while a repulsive interaction impedes it.

It is noted in Figures 4 and 5 that the heat of mixing affects the interlayer spacing of the 1st layer in the liquid. We plot the interlayer spacing as a function of the atomic layers away from the interface with the $\mathrm{Ag}$, $\mathrm{Al}$, and $\mathrm{Cd}$ substrates equilibrated at $1000 \mathrm{~K}$, as shown (a) $\mathrm{L}-\mathrm{Al} / \mathrm{S}-\mathrm{Ag}$

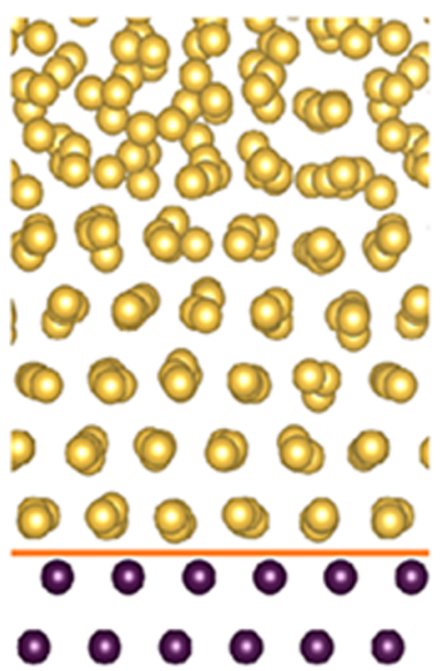

(b) L-Al/S-Al

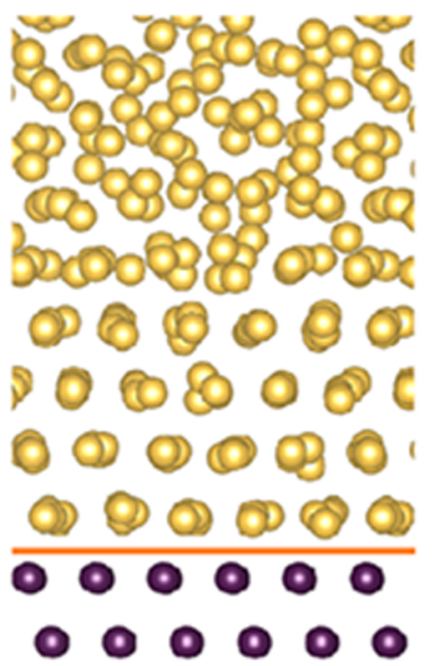

(c) $\mathrm{L}-\mathrm{Al} / \mathrm{S}-\mathrm{Cd}$

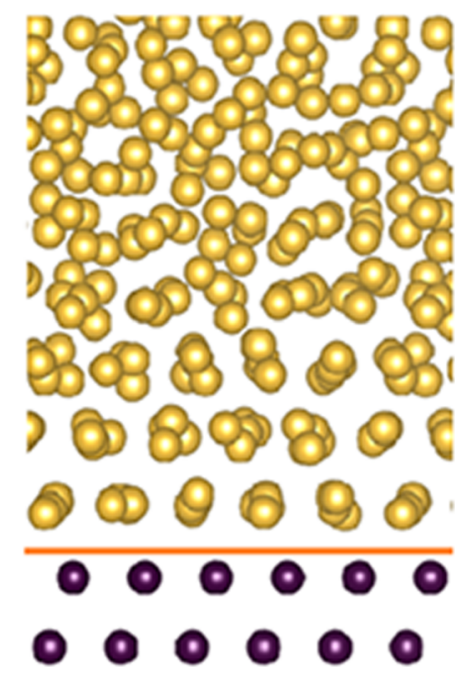

Fig. 3-Snapshots of the simulation systems with the liquid Al and pinned $(a) \mathrm{Ag},(b) \mathrm{Al}$, and $(c) \mathrm{Cd}$ substrates equilibrated at $1000 \mathrm{~K}$. The dark (violet online) and light (gold online) spheres represent the substrate and liquid atoms, respectively, and the solid (orange online) lines mark the interface between the substrate and the liquid (Color figure online). 


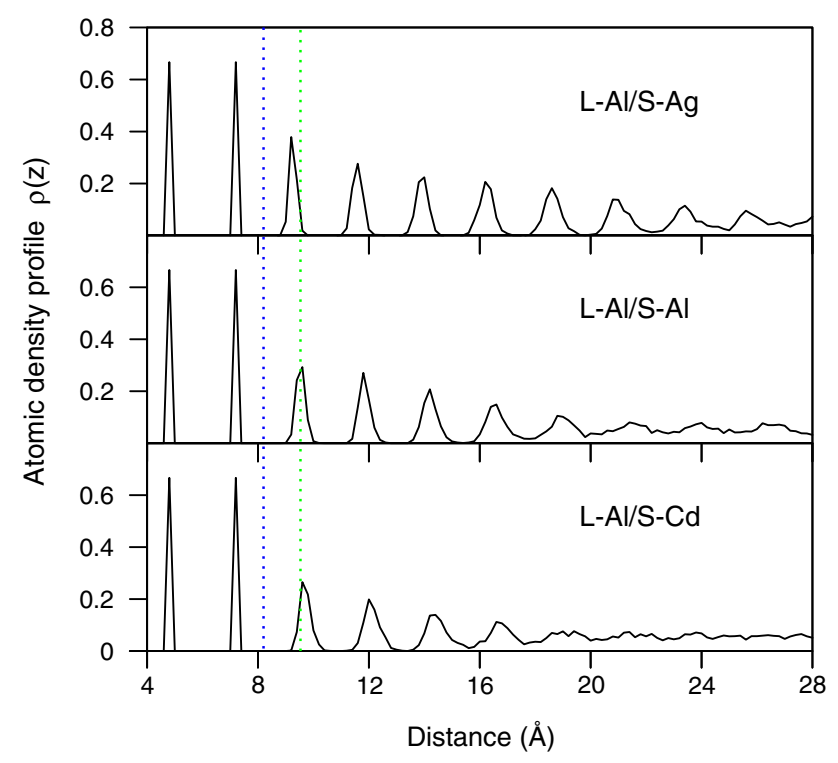

Fig. 4-Atomic density profiles, $\rho(\mathrm{z})$, of the liquid $\mathrm{Al}$ adjacent to $\mathrm{Ag}, \mathrm{Al}$, and $\mathrm{Cd}$ substrates equilibrated at $1000 \mathrm{~K}$. The vertical blue dotted line separates the substrate and liquid, and the green dotted line marks the peak position of the 1st layer of liquid $\mathrm{Al}$ adjacent to the interface (Color figure online).

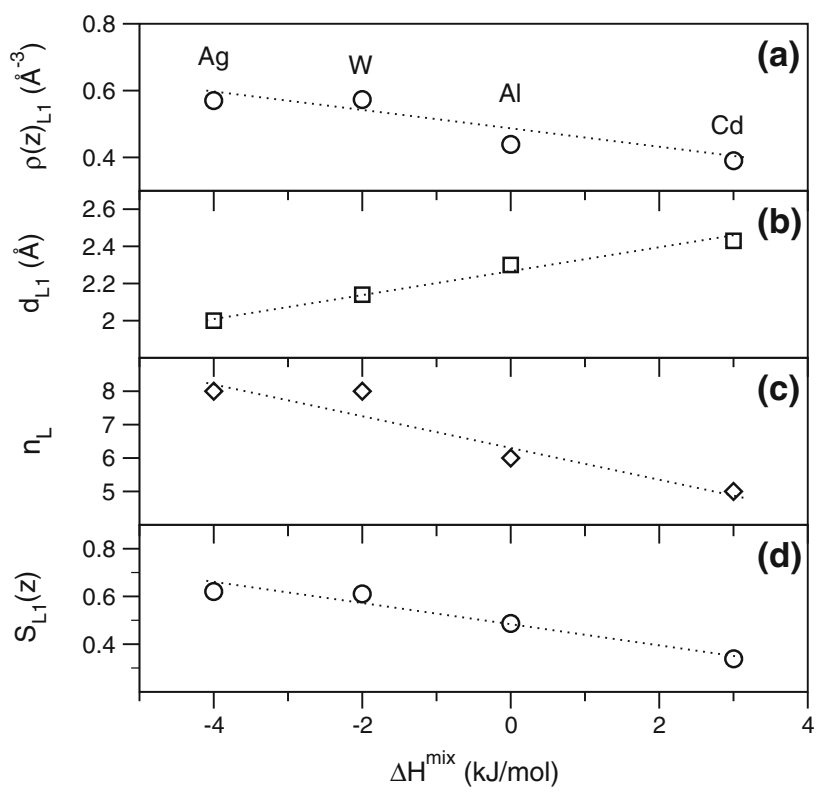

Fig. 5- (a) Peak density, $\rho_{\mathrm{L} 1}(z),(b)$ interlayer spacing, $d_{\mathrm{L} 1}$, of the 1st layer in the liquid, $(c)$ number of layers, $n_{\mathrm{L}}$, at the interface, and $(d)$ in-plane order parameter, $S_{\mathrm{L} 1}(z)$, of the 1st layer as a function of the heat of mixing, $\Delta H^{\text {mix }}$, between the liquid $\mathrm{Al}$ and the substrate element for the systems with pinned substrates equilibrated at $1000 \mathrm{~K}$.

in Figure 6. The interlayer spacing remains more or less constant in the case of the Al substrate, very close to the $\{111\}_{\mathrm{Al}}$ plane spacing at its melting point (solid line in Figure 6). On the other hand, the interlayer spacing of the 1st layer, $d_{\mathrm{L} 1}$, is smaller with the $\mathrm{Ag}$ substrate and larger with the $\mathrm{Cd}$ substrate, while beyond the first layer the interlayer spacing is almost the same as that of the Al substrate regardless of the nature of the substrate.

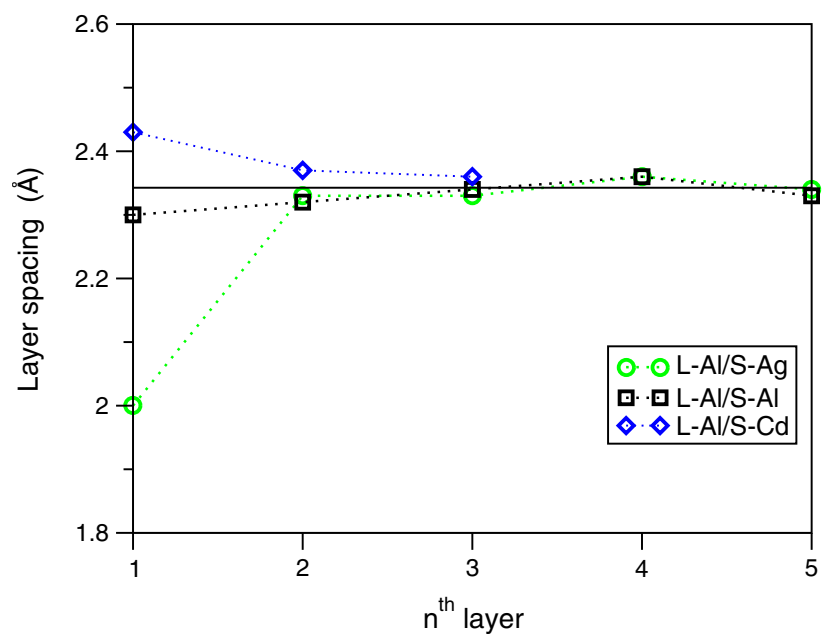

Fig. 6-Interlayer spacing, $d_{\mathrm{L}}$, of $\mathrm{Al}$ atoms in the liquid adjacent to the interface as a function of the atomic layer away from the interface, with pinned $\mathrm{Ag}, \mathrm{Al}$, and $\mathrm{Cd}$ substrates equilibrated at $1000 \mathrm{~K}$. For comparison, the calculated interplanar spacing of (111) plane of fcc $\mathrm{Al}, d_{\{111\} \mathrm{Al}},(2.35 \AA)$ at its melting point is also shown (solid line) (Color figure online).

The $d_{\mathrm{L} 1}$ is $2.01,2.30$, and $2.43 \AA$, respectively, for the pinned $\mathrm{Ag}, \mathrm{Al}$, and $\mathrm{Cd}$ substrates, and decreases linearly with decreasing $\Delta H^{\text {mix }}$, as shown previously in Figure 5(b). Thus, it can be concluded that the attractive interaction between the liquid and the substrate draws the 1st layer closer to the substrate, while a repulsive interaction pushes the 1st layer further away from the substrate.

\section{Chemical Effect on In-Plane Ordering}

The in-plane atomic ordering in the liquid adjacent to the interface was quantified by the in-plane order parameter $S(z)$. Figure 5(d) shows the in-plane order parameter for the 1st layer in the liquid adjacent to the interface, $S(z)_{\mathrm{L} 1}$, as a function of $\Delta H^{\mathrm{mix}}$ for the simulation systems with different substrates equilibrated at $1000 \mathrm{~K}$. In-plane atomic ordering increases with decreasing $\Delta H^{\mathrm{mix}}$ from positive to negative. For example, the $S(z)_{\mathrm{L} 1}$ values for the 1 st layer are $0.34,0.49$, and 0.62 , respectively, for the systems with $\mathrm{Cd}, \mathrm{Al}$, and $\mathrm{Ag}$ substrates, which have a decreasing heat of mixing from $+3 \mathrm{~kJ} / \mathrm{mol}$ for $\mathrm{Cd}$ to $-4 \mathrm{~kJ} / \mathrm{mol}$ for Ag. The quantified in-plane order parameters for all layers are plotted in Figure 7 as a function of the positions of the atomic layers for the pinned $\mathrm{Ag}, \mathrm{Al}$, and $\mathrm{Cd}$ substrates equilibrated at $1000 \mathrm{~K}$. Substantial in-plane ordering persists within the first 4,3 , and 2 atomic layers in the liquid adjacent to the $\mathrm{Ag}, \mathrm{Al}$, and $\mathrm{Cd}$ substrates, respectively. In addition, for a given atomic layer in the liquid at the interface, $S(z)$ always has a higher value for the system with the Ag substrate, a lower value for the system with the Cd substrate, compared with that for the system with the Al substrate.

We have also examined atomic arrangement in the first 5 interfacial layers using the time-averaged atomic positions for the simulation systems with the pinned $\mathrm{Ag}$, $\mathrm{Al}$, and $\mathrm{Cd}$ substrates equilibrated at $1000 \mathrm{~K}$, as shown 
in Figure 8. The atomic arrangement in the liquid adjacent to the interface exhibits a visibly ordered structure within the first 4, 3, and 2 atomic layers, respectively, for the $\mathrm{Ag}, \mathrm{Al}$, and $\mathrm{Cd}$ substrates, a mixed structure with ordered and disordered regions in the following few layers and a disordered structure further away from the interface. Figure 8 also reveals that the atoms in the ordered regions vibrate around their equilibrium lattice sites, while the atoms in the disordered regions move more randomly, in good agreement with our previous large-scale MD simulations. ${ }^{[6]}$

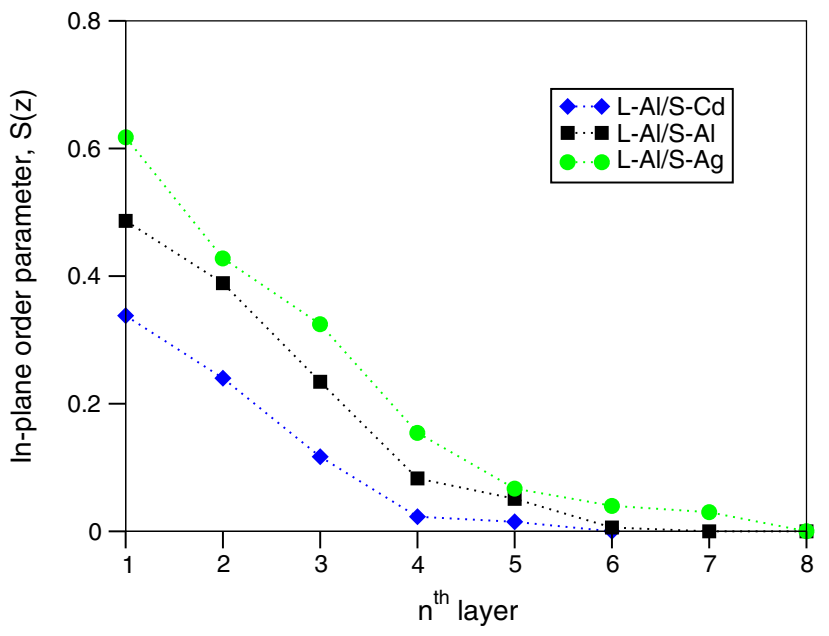

Fig. 7-In-plane order parameter, $S(z)$, of the liquid $\mathrm{Al}$ atoms as a function of the atomic layer away from the interface, with the pinned $\mathrm{Ag}, \mathrm{Al}$, and $\mathrm{Cd}$ substrates equilibrated at $1000 \mathrm{~K}$ (Color figure online).
The time-averaged atomic positions represent the trajectory of an individual atom over a given period of simulation time, which has a projection on a reference plane parallel to the interface, as shown in Figure 8. Thus, the area of the trajectory projection of an individual atom can be taken qualitatively as an indicator of its mobility. Figure 8 suggests that atomic mobility of the liquid adjacent to the interface increases with increasing distance from the interface for a given liquid/substrate system, and increases with increasing heat of mixing for different liquid/substrate systems.

Overall, it can be concluded that in-plane atomic ordering in the liquid is enhanced by substrates having an attractive interaction with the liquid (negative $\Delta H^{\text {mix }}$ ), while it is impeded by substrates having a repulsive interaction with the liquid (positive $\Delta H^{\text {mix }}$ ).

\section{Temperature Effect on Atomic Ordering}

To evaluate the temperature effect, we choose the simulation system of liquid $\mathrm{Al} /$ pinned $\mathrm{Al}$ substrate, for which lattice misfit is 0 pct and $\Delta H^{\mathrm{mix}}$ is 0 . Therefore, the effects of lattice misfit and substrate chemistry on the atomic ordering are excluded. The bulk liquid Al is disordered in the simulation system equilibrated at $3000 \mathrm{~K}$, and is taken as the initial state of the liquid for further simulations. When the liquid is brought into contact with the pinned $\mathrm{Al}$ substrate, the atoms in the liquid become layered at the interface, and the atomic layering increases with decreasing temperature from $2000 \mathrm{~K}$ to $1000 \mathrm{~K}$ (Figures 9(a) through (d)). Figure 10 presents the quantified density profiles, $\rho(\mathrm{z})$, in the liquid as a function of distance $z$ from the interface
1st layer
2nd layer
3rd layer
4th layer
(a)

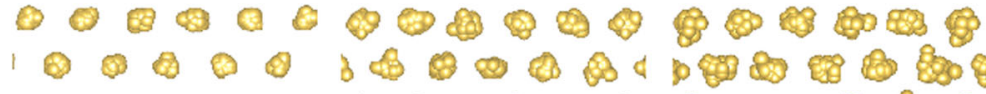

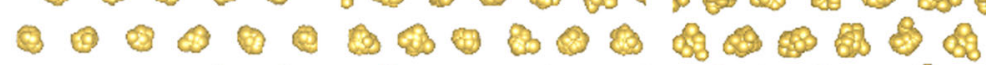

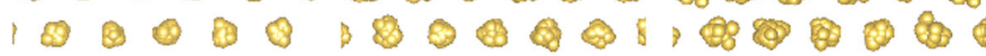

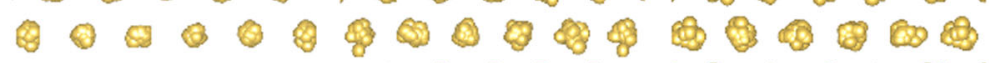

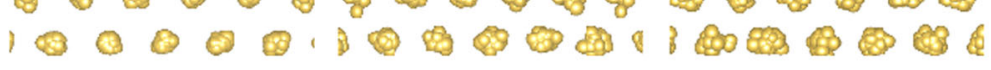

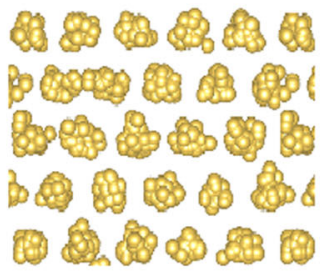

5th layer

(b)
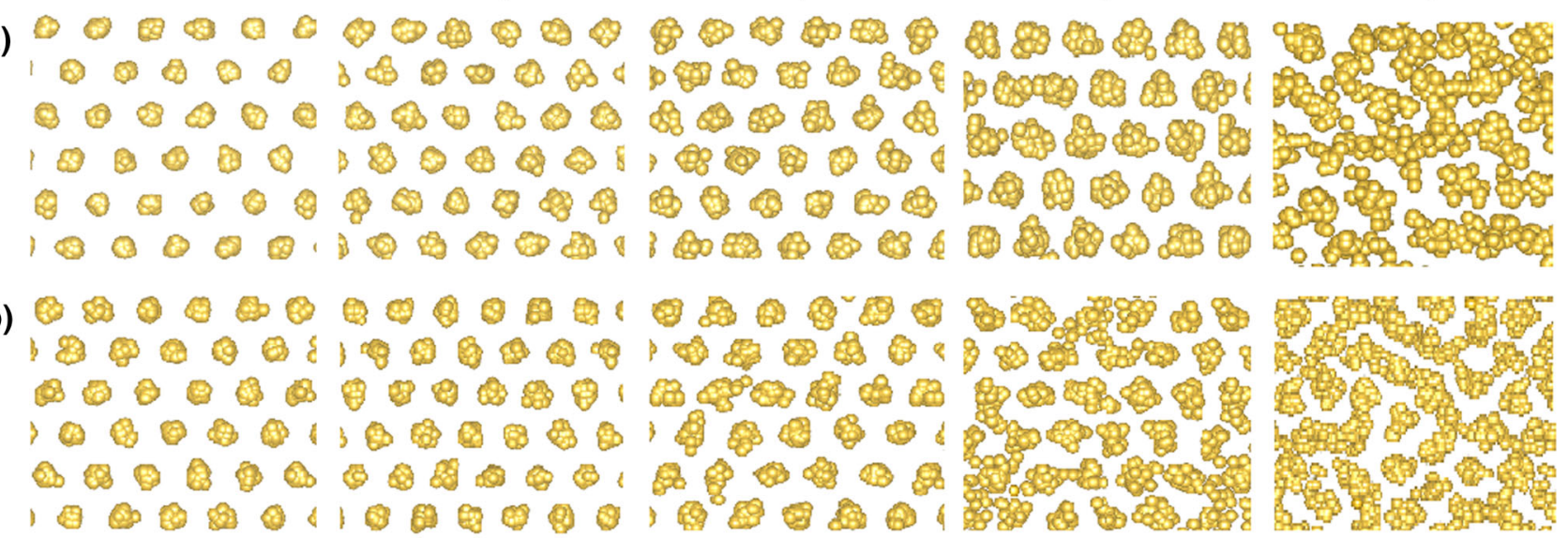

(c)
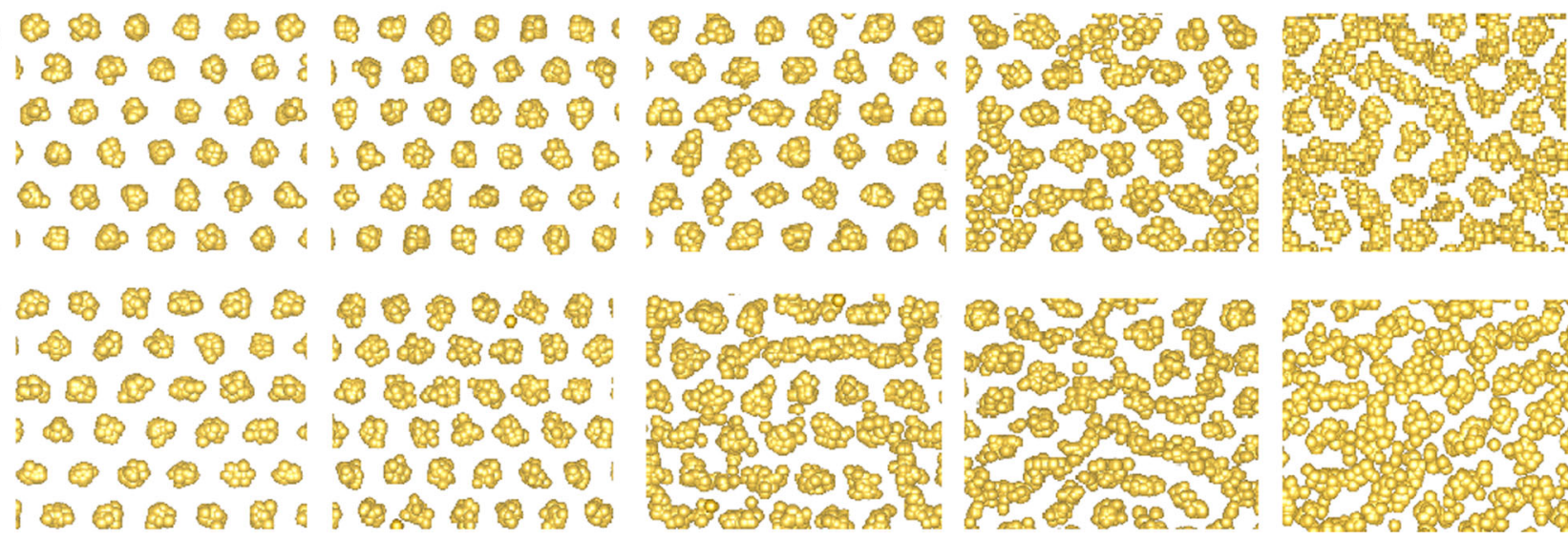

Fig. 8-Time-averaged atomic positions in the first 5 interfacial layers of liquid $\mathrm{Al}$ with (a) pinned $\mathrm{Ag}$, (b) $\mathrm{Al}$, and (c) $\mathrm{Cd}$ substrates equilibrated at $1000 \mathrm{~K}$ (Color figure online). 
equilibrated at various temperatures. The vertical dotted line marks the interface between the substrate and the liquid. It is noted that the layering still persists within $\sim 3$ atomic layers even at $2000 \mathrm{~K}$ (Figure 10(a)). The number of layers adjacent to the interface increases gradually with decreasing temperature, reaching 6 layers at $950 \mathrm{~K}$ (Figure 10(e)). The quantified in-plane order parameter, $S(z)$, of the liquid adjacent to the interface is shown in Figure 11 as a function of atomic layer positions and equilibration temperature. The in-plane ordering adjacent to the interface increased with decreasing temperature. At $2000 \mathrm{~K}$, there is no noticable in-plane atomic ordering in the liquid adjacent to the interface, although layering persists within the first 3 atomic layers (Figure 10(a)). With decreasing temperature, the in-plane ordering extends gradually into further layers in the liquid away from the interface. Based on these results, we can conclude that atomic ordering in the liquid adjacent to a crystalline substrate is enhanced with decreasing temperature.

\section{E. Electron Distribution and Charge Transfer at the Interface}

To understand the effect of substrate chemistry, we examined electronic density distribution of atoms adjacent to the liquid/substrate interface. Figure 12 shows iso-surfaces of electronic density distributions at the interface for the simulation systems of the liquid $\mathrm{Al}$ in (a) $2000 \mathrm{~K}$

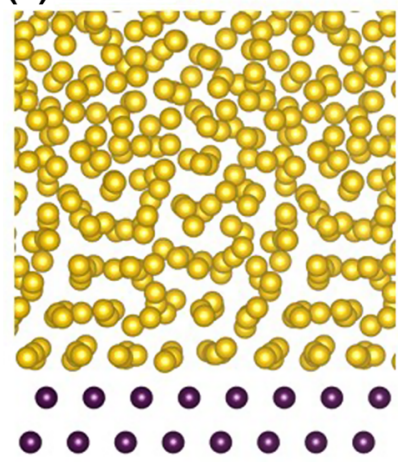

(c) $1200 \mathrm{~K}$

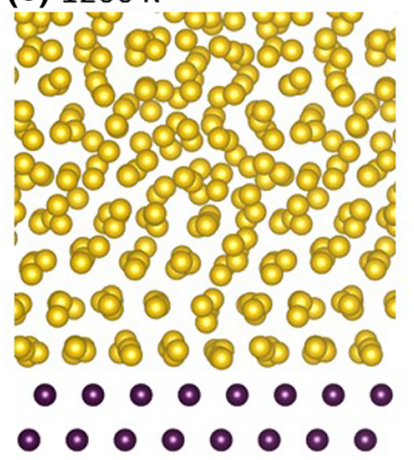

(b) $1500 \mathrm{~K}$

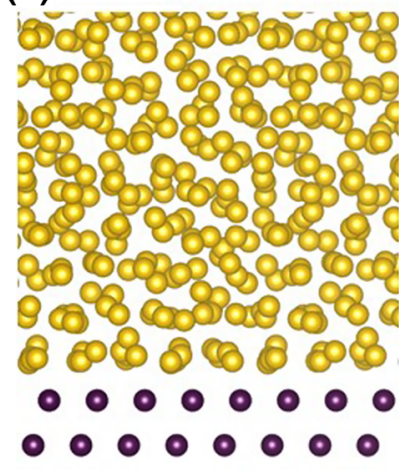

(d) $1000 \mathrm{~K}$

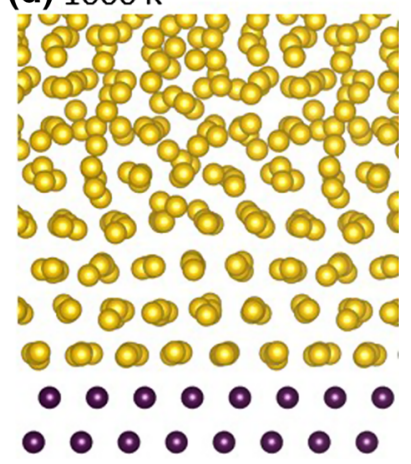

Fig. 9-Snapshots of the simulation systems with liquid $\mathrm{Al}$ and the pinned Al substrate, equilibrated at $(a) T=2000 \mathrm{~K},(b) 1500 \mathrm{~K},(c)$ $1200 \mathrm{~K}$, and (d) $1000 \mathrm{~K}$. The light (gold online) and dark (violet online) spheres represent liquid $\mathrm{Al}$ and substrate atoms, respectively (Color figure online). contact with the pinned ( $\mathrm{Ag}$ and $\mathrm{Cd}$ ) substrates equilibrated at $1000 \mathrm{~K}$. There is higher electron density at the liquid/Ag substrate interface than that at the liquid/Cd substrate interface. This is consistent with the fact that there exists an attractive interaction between $\mathrm{Al}$ and $\mathrm{Ag}$ and a repulsive interaction between the $\mathrm{Al}$ and $\mathrm{Cd}$.

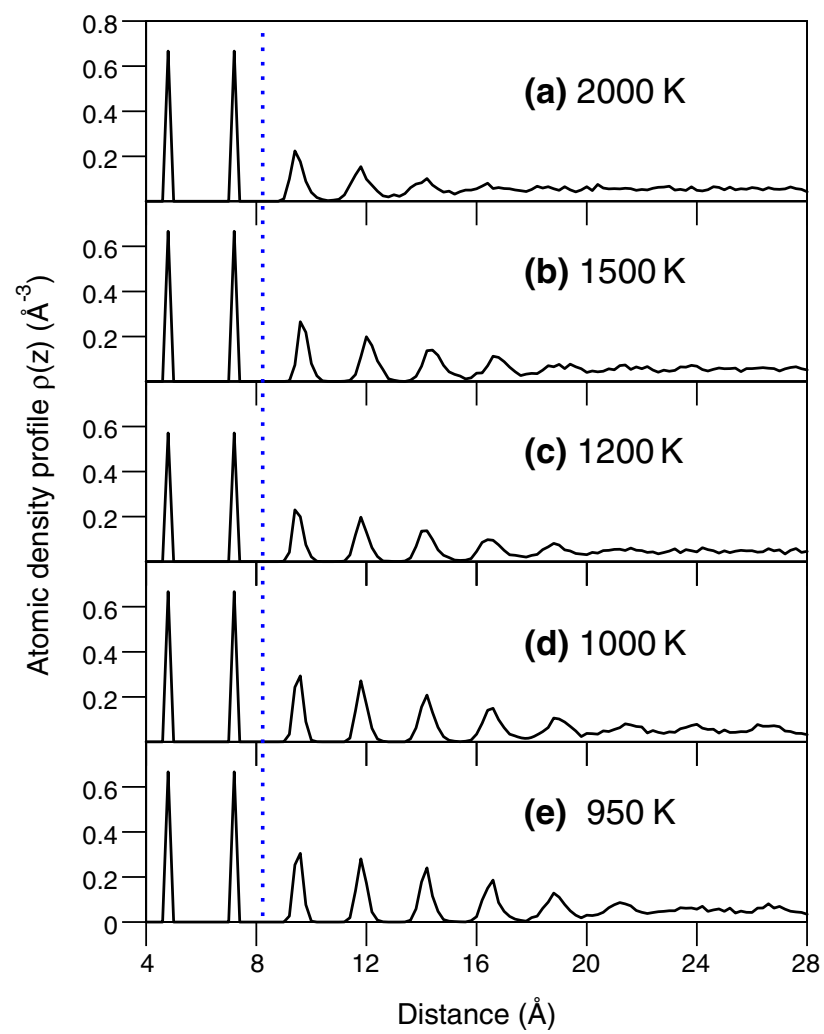

Fig. 10-Atomic density profiles, $\rho(z)$, of the liquid $\mathrm{Al}$ atoms adjacent to the pinned $\mathrm{Al}$ substrate equilibrated at $(a) T=2000 \mathrm{~K}$, (b) $1500 \mathrm{~K},(c) 1200 \mathrm{~K},(d) 1000 \mathrm{~K}$, and (e) $950 \mathrm{~K}$. The vertical blue dotted line marks the interface (Color figure online).

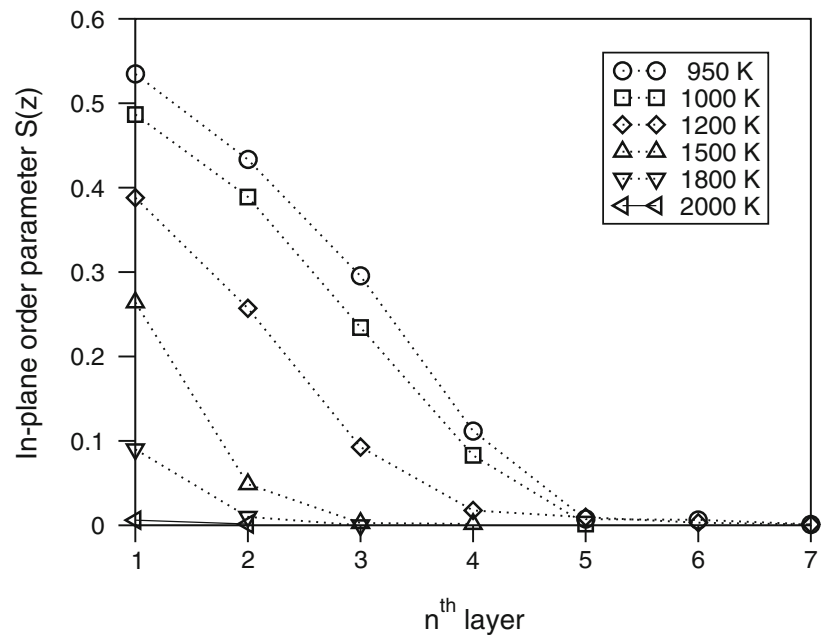

Fig. 11-The in-plane order parameter, $S(z)$, of the liquid $\mathrm{Al}$ atoms adjacent to the interface as a function of atomic layer from the interface in the system with the pinned $\mathrm{Al}$ substrate equilibrated at temperatures from $950 \mathrm{~K}$ to $2000 \mathrm{~K}$. 


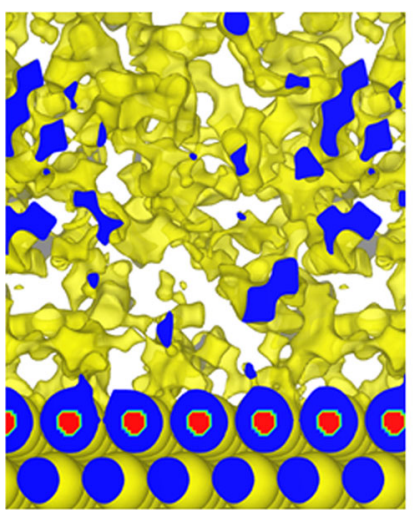

(a)

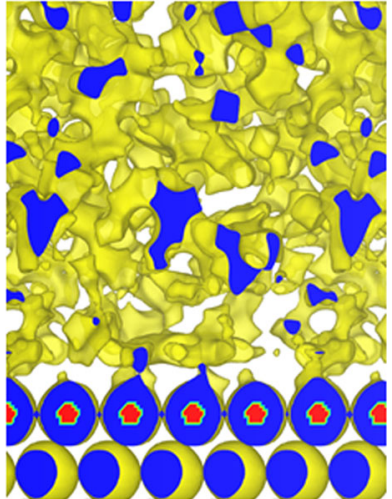

(b)
Fig. 12-Iso-surfaces of electronic density distributions in the simulation systems with the liquid $\mathrm{Al}$ and pinned $(a) \mathrm{Ag}$ and $(b) \mathrm{Cd}$ substrates equilibrated at $1000 \mathrm{~K}$. The yellow color represents the iso-surfaces with electron density $\rho(r)=0.033 \mathrm{e} / \AA^{3}$, the blue for regions with higher electron densities and white for regions of lower densities, while the red regions represent the high density distributions of the $\mathrm{Ag} 4 \mathrm{~d}$ (a) and $\mathrm{Cd} \mathrm{4d} \mathrm{(b)} \mathrm{electrons} \mathrm{around} \mathrm{the}$ atomic sites of the substrates (Color figure online).

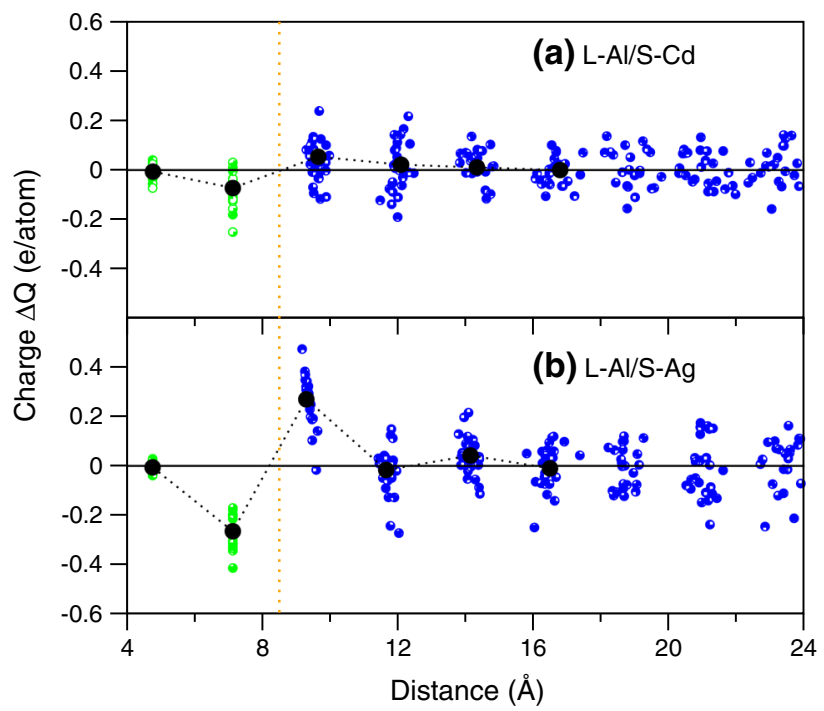

Fig. 13-Charges of the individual atoms (green and blue dots for the substrate and liquid $\mathrm{Al}$ atoms, respectively, online) as a function of distance $z$ in the simulation systems with the liquid $\mathrm{Al}$ and pinned (a) $\mathrm{Cd}$ or (b) $\mathrm{Ag}$ substrates equilibrated at $1000 \mathrm{~K}$. The averaged charge per atom (filled circles, black online) in the individual layer is also included. The dashed (yellow online) vertical line marks the interface (Color figure online).

The charge transfer between the liquid and the substrate across the interface was also investigated, using the method developed by Bader ${ }^{[35]}$ that has been successfully applied to many systems. ${ }^{[33,34]}$ Figure 13 shows the charges of the individual atoms adjacent to the liquid/substrate interface as a function of distance $z$ for the simulation systems of liquid $\mathrm{Al}$ on pinned $\mathrm{Cd}$ and $\mathrm{Ag}$ substrates equilibrated at $1000 \mathrm{~K}$. The data for the average charge per atom of the individual layer are also included. The dashed line marks the interface. The charge transfer between the 1st layer in the liquid and the surface layer of the substrate for the system with the Ag substrate is significantly larger than that with the $\mathrm{Cd}$ substrate. The average charge transfer per atom from the 1st layer in the liquid to the surface layer of the substrate is 0.3 and 0.1 electrons, respectively, for the $\mathrm{Al} / \mathrm{Ag}$ and $\mathrm{Al} / \mathrm{Cd}$ systems. Therefore, it can be concluded that the average charge transfer per atom from the 1st layer in the liquid to the surface layer of the substrate increases with decreasing heat of mixing, i.e., from repulsive to attractive interactions.

\section{DISCUSSIONS}

It is generally believed that atomic ordering in the liquid adjacent to the liquid/substrate interface is affected by both the physical and the chemical nature of the substrate for a given metallic liquid..$^{[2,3,13,36]} \mathrm{We}$ have developed a unique approach to investigate independently the individual effects of chemical or physical properties of the substrate on such atomic ordering. In this approach, the atoms of the substrate are pinned at the equilibrium positions (of Al, in this work) during the simulation, and the liquid is brought into contact with the substrate. To investigate the effect of substrate structure, the atoms of the substrate are chosen to be the same as those of the liquid, while the physical properties of the substrate can be varied systematically, such as crystal structure, surface orientation, surface roughness, and lattice misfit between the substrate and the solid phase. ${ }^{[6,37]}$ In this way, the effect of substrate chemistry on atomic ordering in the liquid at the interface can be eliminated. In the present work, in order to investigate the effect of substrate chemistry, we constructed substrates artificially, which have the same physical nature (crystal structure, surface orientation, and lattice parameter) as that of the solid phase, but exhibit different chemical characteristics by pinning atoms of a selected element at the equilibrium positions of the substrate. By doing so, the effect of substrate structure on atomic ordering can be successfully excluded. In addition, we use heat of mixing $\left(\Delta H^{\mathrm{mix}}\right)$ as a measure to quantify the chemical interaction between the liquid and the substrate: a large and negative $\Delta H^{\text {mix }}$ suggests a strong attractive chemical interaction; a large and positive $\Delta H^{\text {mix }}$ indicates a strong repulsive chemical interaction; and $\Delta H^{\text {mix }}=0$ means no chemical interaction. By selecting suitable elements to construct substrates, we are able to simulate atomic ordering in the liquid at the interface with a wide range of chemical interactions (See Table I).

The validity of this approach has been confirmed by ab initio MD simulations in this work and classical MD simulations presented elsewhere. ${ }^{[7]}$ With ab initio MD simulation, this study revealed that the pinned and relaxed substrates produce only minor difference in atomic ordering in the liquid at the interface, suggesting that the pinned substrate approach would not affect the general trend of atomic ordering (Figures 1 and 2). This study also revealed that the $\mathrm{Al}$ atoms in the liquid adjacent to a pinned Al substrate exhibit pronounced atomic ordering at temperatures above the nucleation 
temperature, including both atomic layering and in-plane atomic ordering (Figures 3(b), 4 and 7). These results are in good agreement with that obtained from large-scale MD simulations. ${ }^{[7]}$ This suggests that this approach is a reliable method to obtain the fundamental information of atomic ordering in the liquid adjacent to the liquid/substrate interface, and therefore can be used to investigate the effect of substrate chemistry on the atomic ordering in the liquid adjacent to the interface.

Both chemistry and structure of the substrate can affect atomic ordering in the liquid at the interface. With our new approach, for the first time, we have assessed individually the effects of the substrate chemistry and structure on atomic ordering in the liquid adjacent to the interface. Our previous MD simulations ${ }^{[16]}$ revealed that a smooth crystalline surface can induce significant atomic ordering in the liquid adjacent to the interface, which is manifested by atomic layering normal to the interface, in-plane atomic ordering parallel to the interface, and the formation of a $2 \mathrm{D}$ ordered structure at the interface through a structural templating mechanism. More importantly, we found that such atomic ordering in the liquid can be significantly enhanced by decreasing the lattice misfit between the substrate and the solid phase corresponding to the liquid upon solidification, ${ }^{[14]}$ and impeded or even eliminated by increasing the atomic level surface roughness of the substrate. ${ }^{[15,37]}$ The effects of both lattice misfit and atomic surface roughness on atomic ordering in the liquid adjacent to the interface can be attributed to the increased atomic mobility in the liquid adjacent to the interface, which in turn weakens the structural templating for atomic ordering in the liquid.

In the present work, using this unique approach we have successfully investigated the effect of substrate chemistry on atomic ordering in the liquid adjacent to the liquid/substrate interface by excluding the structural effect. Our $a b$ initio MD simulations revealed that an attractive chemical interaction between the liquid and the substrate (negative $\Delta H^{\mathrm{mix}}$ ) increases atomic ordering in the liquid at the interface, while a repulsive chemical interaction (positive $\Delta H^{\mathrm{mix}}$ ) impedes such atomic ordering (Figures 5, 7 and 8). The attractive interaction leads to a reduced interlayer spacing between the 1st layer in the liquid and the surface layer of the Ag substrate, compared with that for the Al substrate (Figure 6), and therefore strengthens the structural templating through increased bond strength. On the other hand, the repulsive interaction leads to a larger interlayer spacing between the 1st layer in the liquid and the surface layer of the $\mathrm{Cd}$ substrate, and hence weakens the structural templating. Therefore, it can be concluded that an attractive interaction between the liquid and the substrate strengthens prenucleation, while a repulsive interaction weakens it.

The effect of substrate chemistry on the atomic ordering in the liquid adjacent to the interface can be related to the charge transfer between the substrate and the liquid. The electronic density at the interface for the system with the Ag substrate is higher than that with the Cd substrate (Figure 12), suggesting a stronger bonding at the interface between $\mathrm{Al}$ and $\mathrm{Ag}$ atoms than that between $\mathrm{Al}$ and $\mathrm{Cd}$ atoms. There is a larger average charge transfer of 0.3 electrons per atom from the Al atoms in the 1st layer in the liquid to the Ag atoms in the surface layer of the substrate, compared to 0.1 electrons per atom from the $\mathrm{Al}$ atoms to the $\mathrm{Cd}$ atoms at the interface (Figure 13). This is consistent with the difference in the electronegativity between the relevant elements, which is 1.61 for $\mathrm{Al}, 1.69$ for $\mathrm{Cd}$, and 1.93 for Ag in Pauling scale. However, it should be pointed out that the charge transfer only occurs between the 1 st layer in the liquid and the surface layer of the substrate. Consequently, the change in the interlayer spacing is only observed between the 1st layer in the liquid and the surface layer of the Ag and Cd substrates, while the interlayer spacing remains constant further away from the interface, which is equal to the $\{111\}_{\mathrm{Al}}$ plane spacing (Figure 6). It is well understood that chemical bond strength between two atoms/ions decays exponentially with the bond length and exists mainly between the nearest neighboring atoms. ${ }^{[35,38]}$ Therefore, the chemical effect of the substrate is expected to be local and is mainly confined to atoms in the 1st layer of the liquid; such effect can be ignored beyond the 1st layer of the liquid.

It is important to realize that although both structure and chemistry of the substrate affect atomic ordering in the liquid adjacent to the liquid/substrate interface, the structural effect dominates the atomic ordering process while the chemical effect is only a secondary factor. Because the chemical effect can only be realized through structural templating, no chemical effect exists in the absence of structural templating. The physical origin of atomic ordering in the liquid adjacent to a substrate is structural templating, in which the crystal lattice in the substrate surface provides potential low-energy positions for the formation of ordered structures in the $1 \mathrm{st}$ layer in the liquid, which in turn provides a template for the formation of ordered structures in the 2nd layer, and so on. Substrate chemistry affects atomic ordering through either strengthening or weakening the structural templating. As demonstrated in a previous study, ${ }^{[37]}$ there is no atomic ordering in the liquid adjacent to a bulk amorphous substrate. This means that there is no atomic ordering in the liquid at the interface without structural templating. For a given liquid, an attractive substrate strengthens structural templating and hence increases atomic ordering, while a repulsive substrate weakens structural templating and thus impedes atomic ordering.

In this study, we found that atomic ordering in the liquid adjacent to the liquid/substrate interface decreases with increasing temperature (Figures 10 and 11). Similar to the substrate chemistry, temperature affects the atomic ordering through its influence on structural templating. Increasing temperature increases thermal vibration of the atoms in the liquid due to the increased kinetic energy, which weakens the structural templating and consequently reduces atomic ordering in the liquid. The effect of thermal vibration on the atomic ordering has been demonstrated in Figures 1 and 2, which shows that reducing thermal vibration by pinning the substrate atoms at the equilibrium positions 
increases the atomic ordering in the liquid. Alternatively, the temperature effect can be understood in terms of thermal expansion. Increasing temperature increases the average atomic bond length (thermal expansion) and consequently decreases atomic bond strength between the 1st layer of the liquid and the surface layer of the substrate, resulting in a lower efficiency for structural templating by the substrate. This is similar to the effect of substrate chemistry as discussed previously. Therefore, temperature is another secondary factor for atomic ordering; it affects the atomic ordering through either strengthening or weakening the efficiency of structural templating induced by the substrate.

From the viewpoint of heterogeneous nucleation, atomic ordering in the liquid adjacent to the liquid/substrate interface at temperatures above nucleation temperature has been referred to as prenucleation, which can be taken as the precursor for heterogeneous nucleation. ${ }^{[14]}$ The epitaxial nucleation model ${ }^{[3]}$ suggests that heterogeneous nucleation proceeds through layerby-layer growth by a structural templating mechanism, starting with a 2D ordered structure induced by the substrate. Therefore, structural templating is the essential process for both prenucleation and heterogeneous nucleation. Fundamentally, structural templating can be either enhanced by reducing the lattice misfit between the substrate and the solid phase, or impeded by increasing the lattice misfit. ${ }^{[14]}$ In addition, this work has provided further scientific understanding: structural templating can be either strengthened by a substrate that has an attractive interaction with the liquid (negative $\Delta H^{\mathrm{mix}}$ ), or weakened by a substrate that has a repulsive interaction with the liquid (positive $\Delta H^{\mathrm{mix}}$ ).

Practically, this work also provides useful insight on grain refinement. In the current industrial practice of casting of Al alloys, for example, grain refinement is usually achieved either by ex situ particles (e.g., $\left.\mathrm{TiB}_{2}\right)$ through chemical inoculation, ${ }^{[39]}$ or by in situ particles (e.g., oxides). ${ }^{[40]}$ In the case of chemical inoculation, $\mathrm{TiB}_{2}$ has a Ti-terminated (0001) surface and a relatively large lattice misfit with $\alpha$-Al (4.2 pct), suggesting that $\mathrm{TiB}_{2}$ is not a potent substrate for heterogeneous nucleation of $\alpha$-Al. Through the strong attractive interaction with liquid $\mathrm{Al}$ containing $\mathrm{Ti}$, a (112) $\mathrm{Al}_{3} \mathrm{Ti}$ 2-dimensional compound (2DC) is formed on the (0001) $\mathrm{TiB}_{2}$ surface during the production of the grain refiner, which reduces the lattice misfit between the substrate and the solid from -4.2 to 0.09 pet, making such $\mathrm{Al}_{3} \mathrm{Ti}$ 2DC-coated $\mathrm{TiB}_{2}$ particles extremely potent for heterogeneous nucleation of $\alpha$-Al and the Al-Ti-B-based grain refiners very effective for grain refinement of most of $\mathrm{Al}$ alloys. ${ }^{[41]}$ However, in $\mathrm{Zr}$-containing $\mathrm{Al}$ alloys, the $\mathrm{Al}_{3} \mathrm{Ti} 2 \mathrm{DC}$ becomes thermodynamically unstable and dissolves into the liquid. The strong attractive interaction between the naked $\mathrm{TiB}_{2}$ surface and the solute $\mathrm{Zr}$ results in the formation of a $\mathrm{Ti}_{2} \mathrm{Zr} 2 \mathrm{DC}$ to replace the original Ti-terminated surface. This gives rise to a significant increase of lattice misfit from 0.09 to -4.7 pct, rendering such $\mathrm{Ti}_{2} \mathrm{Zr} 2 \mathrm{DC}$-terminated $\mathrm{TiB}_{2}$ particles impotent for heterogeneous nucleation and Al-Ti-Bbased grain refiners ineffective for grain refinement of Zr-containing Al alloys. ${ }^{[42]}$
In the case of in situ grain refinement, metal oxides usually have O-terminated surfaces since the building block for metal oxides is a tetrahedron with 4 oxygen atoms at its corners and 1 metal atom at its center. The O-terminated oxide surface has a very strong attractive interaction with the liquid alloys, leading to a strong chemical segregation at the liquid/substrate interface. Such chemical segregation can be positively manipulated to either enhance heterogeneous nucleation by reducing the lattice misfit, or to impede heterogeneous nucleation by increasing the lattice misfit. By appropriate choice of the substrate and trace element addition in the liquid, it is possible to develop self-grain-refining alloys, which is very desirable for the development of closed-loop recyclable alloys.

\section{SUMMARY}

Both crystal structure and chemistry of the substrate affect atomic ordering in the liquid adjacent to the liquid/substrate interface. In this study, we have developed a unique atomistic approach to simulate the effect of substrate chemistry without the interference of the substrate structure by construction of artificial substrates that have the same crystal structure but different chemical characteristics. Using $a b$ initio MD simulations, we found that for a given liquid, an attractive chemical interaction (a negative heat of mixing) between the liquid and the substrate strengthens atomic ordering in the liquid at the interface, while a repulsive interaction (a positive heat of mixing) weakens atomic ordering. This effect of substrate chemistry on the atomic ordering can be attributed to its influence on structural templating by the substrate. An attractive chemical interaction leads to a higher electron density at the interface and a higher electron transfer between the liquid and the substrate across the interface, which in turn reduces the atomic mobility of the atoms in the liquid adjacent to the liquid/substrate interface, and therefore strengthens the structural templating for atomic ordering in the liquid at the interface. It is realized that although both structure and chemistry of the substrate affect atomic ordering in the liquid adjacent to the liquid/substrate interface, the structural effect dominates the atomic ordering process while the chemical effect is only a secondary factor, since the substrate chemistry affects atomic ordering through either strengthening or weakening the structural templating. In addition, we have also investigated the effect of temperature on the atomic ordering in the liquid adjacent to the liquid/substrate interface by excluding both the structural effect (lattice misfit is 0 pct) and the effect of substrate chemistry (heat of mixing is 0 ). We found that temperature is another secondary factor affecting atomic ordering at the interface; temperature affects the atomic ordering through either strengthening or weakening the efficiency of structural templating induced by the substrate depending on whether temperature is decreased or increased. Such knowledge of atomic ordering may help us to further understand both prenucleation and heterogeneous nucleation and to 
develop more effective approaches for grain refinement during solidification through effective manipulation of the interplay between structural and chemical effects of the substrate on atomic ordering in the liquid at the interface.

\section{ACKNOWLEDGMENTS}

We thank Prof. Alan Dinsdale, Dr. P. Quested, and Dr. Y. Wang (BCAST) for useful discussions. Dr. Ian Stone (BCAST) is acknowledged for critical reading of the manuscript. The financial support of the EPSRC, under Grant EP/N007638/1, is gratefully acknowledged.

\section{OPEN ACCESS}

This article is distributed under the terms of the Creative Commons Attribution 4.0 International License (http://creativecommons.org/licenses/by/4.0/), which permits unrestricted use, distribution, and reproduction in any medium, provided you give appropriate credit to the original author(s) and the source, provide a link to the Creative Commons license, and indicate if changes were made.

\section{REFERENCES}

1. K.F. Kelton and A.L. Greer: Nucleation in Condensed Matter: Applications in Materials and Biology, Pergamon Oxford, 2010.

2. W.D. Kaplan and Y. Kauffmann: Annu. Rev. Mater. Res., 2006, vol. 36 , pp. $1-48$

3. Z. Fan: Metall. Mater. Trans. A, 2013, vol. 44A, pp. 1409-18.

4. S.H. Oh, Y. Kauffmann, C. Scheu, W.D. Kaplan, and M. Rühle: Science, 2005, vol. 310, pp. 661-63.

5. Y. Kauffmann, S.H. Oh, C.T. Koch, A. Hashibon, C. Scheu, M. Rühe, and W.D. Kaplan: Acta Mater., 2011, vol. 59, pp. 4378-86.

6. H. Men and Z. Fan: Comput. Mater. Sci., 2014, vol. 85, pp. 1-7.

7. A. Hashibon, J. Adler, M.W. Finnis, and W.D. Kaplan: Interface Sci., 2001, vol. 9, pp. 175-81.

8. A. Hashibon, J. Adler, M.W. Finnis, and W.D. Kaplan: Comp. Mater. Sci., 2002, vol. 24, pp. 443-52.

9. O.M. Magnussen, B.M. Ocko, M.J. Regan, K. Pershan, and M. Deutsch: Phys. Rev. Lett., 1995, vol. 74, pp. 4444-47.

10. M.J. Regan, E.H. Kawamoto, S. Lee, P.S. Pershan, N. Maskil, M. Deutsch, O.M. Magnussen, B.M. Ocko, and L.E. Berman: Phys. Rev. Lett., 1995, vol. 75, pp. 2498-2501.

11. C.J. Yu, A.G. Richter, A. Datta, M.K. Durbin, and P. Dutta: Phys. Rev. Lett., 1999, vol. 82, pp. 2326-29.
12. M.J. Regan, P.S. Pershan, O.M. Magnussen, B.M. Ocko, M. Deutsch, and L.E. Berman: Phys. Rev. B, 1999, vol. 55, pp. 15874-84.

13. K.F. Kelton, A.L. Greer, D.M. Herlach, and D. Holland-Moritz: MRS Bull., 2004, vol. 12, pp. 940-44.

14. H. Men and Z. Fan: Metall. Mater. Trans. A, 2018, vol. 49A, pp. $2766-77$.

15. P. Geysermans, D. Gorse, and V. Pontikis: J. Chem. Phys., 2000, vol. 113 , pp. 6382-89.

16. J.S. Wang, A. Horsfield, U. Schwingenschlögl, and P.D. Lee: Phys. Rev. B, 2010, vol. 82, p. 184203.

17. A. Takeuchi and A. Inoue: Mater. Trans., 2005, vol. 46, pp. 2817-29.

18. W.B. Pearson: Handbook of Lattice Spacings and Structures of Metals and Alloys, Pergamon Press, Oxford, 1967.

19. S.S. Lim, P.L. Rossiter, and J.E. Tibballs: CALPHAD, 1995, vol. 19, pp. 131-41.

20. L.F. Mondolfo: Aluminium Alloys: Structure and Properties, Butterworth \& Co (Publisher) Ltd, London, 1976, pp. 240-42.

21. I. Ansara, A.T. Dinsdale, and M.H. Rand: COST507, Thermochemical Database for Light Metal Alloys, European Communities, Luxmbourg, 1998, vol. 2.

22. C.M. Fang, H. Men and Z. Fan: Proceedings of the $6^{\text {th }}$ decennial international conference on solidification processing, 2017, July 25-28, pp. 52-55.

23. R.O. Jones: Rev. Mod. Phys., 2015, vol. 87, pp. 897-923.

24. G. Kresse and J. Hafner: Phys. Rev. B, 1994, vol. 49, pp. 14251-69.

25. G. Kresse and J. Furthmüller: Comput. Mater. Sci., 1996, vol. 6, pp. $15-50$.

26. P.E. Blöchl: Phys. Rev. B, 1994, vol. 50, pp. 17953-79.

27. G. Kresse and J. Joubert: Phys. Rev. B, 1999, vol. 59, pp. 1758-75.

28. J.P. Perdew, K. Burke, and M. Ernzerhof: Phys. Rev. Lett., 1996, vol. 77 , pp. 3865-68.

29. C. Amador, W.R.L. Lambrecht, and B. Segall: Phys. Rev. B, 1992, vol. 46, pp. 1870-73.

30. C.M. Fang, M.A. van Huis, M.F.H. Sluiter, and H. Zandbergen: Acta Mater., 2010, vol. 58, pp. 2968-77.

31. H.J. Monkhorst and J.D. Pack: Phys. Rev. B, 1976, vol. 13, pp. 5188-92.

32. L.E. Hintzsche, C.M. Fang, M. Marsman, G. Jordan, M.W.P.E. Lamers, A.W. Weeber, and G. Kresse: Phys. Rev. B, 2013, vol. 88, p. 155204

33. C.M. Fang, W.-F. Li, R. Koster, J. Klimeš, A. van Blaaderen, and M.A. van Huis: Phys. Chem. Chem. Phys., 2015, vol. 17 , pp. $365-75$.

34. J.R. Hook and H.E. Hall: Solid State Physics, 2nd ed., Wiley, Chichester, 1991.

35. R.F.W. Bader: J. Phys. Chem. A, 1998, vol. 102, pp. 7314-23.

36. A.L. Greer: Nat. Mater., 2006, vol. 5, pp. 15-18.

37. B. Jiang, H. Men, and Z. Fan: Comput. Mater. Sci., 2018, vol. 153, pp. 73-81.

38. I.D. Brown: Chem. Rev., 2009, vol. 109, pp. 6858-6919.

39. A.L. Greer: J. Chem. Phys., 2016, vol. 145, p. 211704

40. H.-T. Li, Y. Wang, and Z. Fan: Acta Mater., 2012, vol. 60, pp. 1528-37.

41. Z. Fan, Y. Wang, Y. Zhang, T. Qin, X.R. Zhou, G.E. Thompson, T. Pennycook, and T. Hashimoto: Acta Mater., 2015, vol. 84, pp. 292-304.

42. Y. Wang, L. Zhou, B. Jiang, Z. Fan, T. Hashimoto, X. Zhou and Q.M. Ramasse: Acta Mater., 2018 (accepted). 